\title{
A aggressive osteosarcoma of mandible with lung metastasis in a 5 year child: A case report
}

\section{Govardhan H. B, Satyajit Pradhan, Rashmi Singh, Prakash Swain, Lalit Agarwal}

Departments of Radiotherapy and Radiation Medicine, Banaras Hindu University (BHU), Varanasi, India

\section{Email address:}

hbgovardhan@yahoo.co.in (Govardhan H. B.)

\section{To cite this article:}

Govardhan H. B, Satyajit Pradhan, Rashmi Singh, Prakash Swain, Lalit Agarwal. A Aggressive Osteosarcoma of Mandible with Lung Metastasis in a 5 Year Child: A Case Report. Cancer Research Journal. Vol. 2, No. 2, 2014, pp. 15-17. doi: 10.11648/j.crj.20140202.11

\begin{abstract}
Osteosarcoma is a malignant mesenchymal tumor whose cancerous cells produce osteoid matrix. It is the most common primary malignant bone tumor, accounting for approximately $20 \%$ of the sarcomas, but osteosarcoma of mandible is rare only $5 \%$ of the osteosarcomas occur in the jaws and occur mainly in adult population in their later part of the life. They present various clinical and histological aspects, as well as variable disease progression and outcome. Mandibular osteosarcoma are slow growing tumors and have better prognosis compared to other site. Lung is the most common site of metastasis in osteosarcoma mainly in the long bones, in craniofascial osteosarcoma lung metastasis is a rare findings. We are presenting a case of osteosarcoma of mandible in 5 year child with aggressive initial presentation.
\end{abstract}

Keywords: Aggressive Mandibular Osteosarcoma, Lung Metastasis

Osteosarcoma(OS) is the most common primary malignant bone neoplasm, predominantly occurring in long bones and occasionally in the maxillofacial area. Accounts $5-9 \%$ of OS, Maxilla and the mandible are the most involved site.1-4 Mandibular osteosarcoma is often considered a distinct entity because of its predilection to older patients (mean age: 34 to 36 years). In addition, metastases are rare and the prognosis is significantly better and rarely develops distant metastases, when compared to its counterpart in long bones.3-8 we are presenting a case of 5 year old male child a case of osteosarcoma of mandible with lung metastasis.

\section{Case Report}

A 5 year old male child presented to a dentist with history of spontaneous swelling in right mandible for 6 months. Suspecting an abscess, the dentist managed conservatively with coarse of antibiotics. The swelling continued to increase in size. After one month of conservative management child was landed in Department of Radiotherapy and Radiation Medicine, Institute of Medical Sciences, Banaras Hindu University, Varanasi, India. In our department detail clinical examination, evaluation and management done as follows.

On examination, Single swelling of $7 * 6 \mathrm{~cm}$ present over the right mandibular area, no tender to touch. Intra orally, bulging present over right alveolus with no mucosal breach (Fig. 1). Contrast enhancing CT scan face done shows, a large geographical mixed sclerotic and lytic lesion involving right distal body and ramus of the mandible with few area of cortical breaks, surrounding soft tissue component infiltrating into right infra temporal fossa, right messeter and pterygoid muscle (Fig 2 A. B).
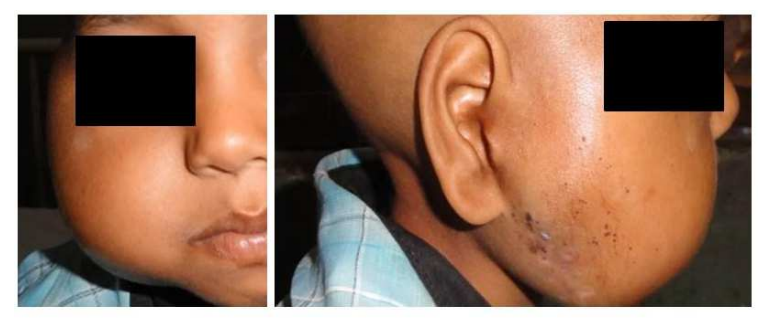

Fig 1: Clinical phrtograph

Fig 1. Clinical photograph of the child showing swelling over right jaw.

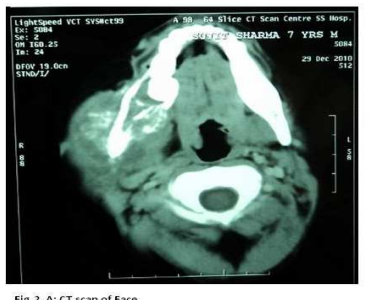

Fig 2 A: CT scan of Face

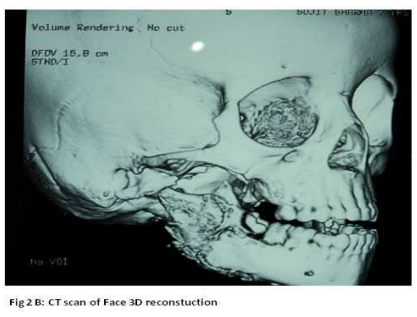

Fig 2B: CT scan of Face 30 reconstuction
Fig 2 A. B. CT Scan face transverse and $3 D$ section. 
Chest $\mathrm{X}$ ray AP view reveals a homogenous well defined round opacity, present in the lower lobe of right lung suggestive of metastasis. (Fig 3 A).CT thorax shows: Homogenous well defined round opacity, present in the lower lobe of right lung and slight pleural effusion, suggestive of metastasis (Fig 3 B ). Biopsy from mandibular swelling lung lesion were taken: in the both the tissue, $\mathrm{H}$ and E stained section shows tumor tissue with individual cell round and spindle shaped cell with moderate amount of cytoplasm and round to spindle nuclei. Also shows large areas of osteoid. Which is eosinophilic and surrounded by these tumor cells suggestive of poorly differentiated osteosarcoma (fig-4). And Immunohistochemistry-CD99 Negative. The Complete hemogram, liver function test, renal function test,
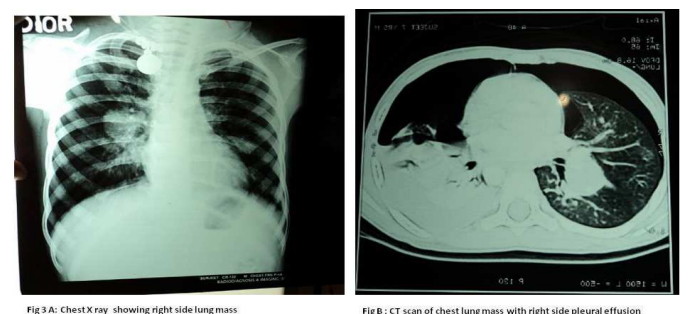

Fig 3A. Chest X ray,B-CT thorax-Showing Lung metastasis.

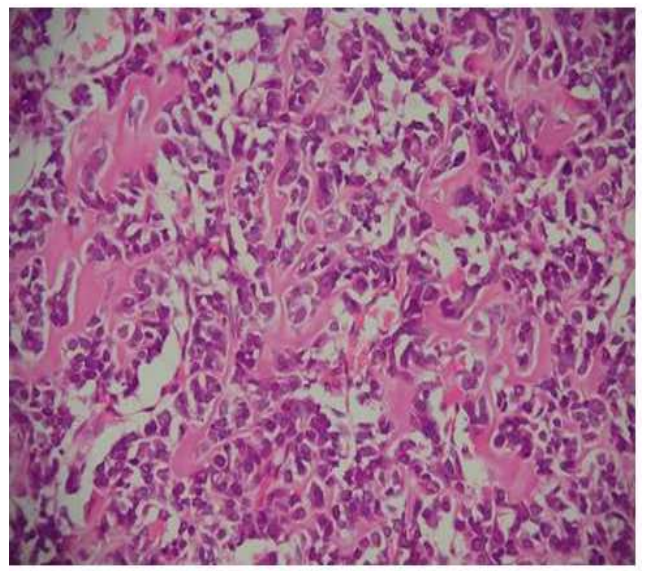

Fig 4. Histology of the mandibular swelling.

Ultrasonography of the abdomen, Bone scan and Bone Marrow Biopsy were within normal limits.

Patient was planned to give 6 cycles of palliative chemotherapy (doxorubicin $25 \mathrm{mg} / \mathrm{m}^{2}$ and cisplatin $100 \mathrm{mg} / \mathrm{m}^{2}$ ). After the 4 cycles of chemotherapy, response evaluation done suggestive of progressive disease (PD). Then the case discussed in the tumor board and planned for $2^{\text {nd }}$ line chemotherapy but care giver were refused and was kept on best supportive care. On telephonic interview child was died after 2 months.

\section{Discussion}

Craniofascial osteosarcoma (CFOS) accounts for $6-10 \%$ of all OS, with mandibular location in approximately $40 \%$ of patients. ${ }^{3-7}$ Several differences in disease characteristics distinguish CFOS and OS at other primary sites: CFOS most commonly affects patients in their $30 \mathrm{~s}$; distant metastases are rarely reported (10-20\% of patients); survival after surgery alone has historically been better for CFOS than for OS arising at other locations, with reported 5-year survival rates of $23-37 \%$ and $10-20 \%$ for CFOS and other OS, respectively. ${ }^{4-9}$

Mardinger et al. ${ }^{10}$ presented 14 cases of OS of the maxilla and conducted their discussion based on a literature review of 774 cases reported in the English literature. Differences between OS of the maxilla and OS of long bones were also analyzed. Patients' ages ranged from 8 to 78 years (mean age of 33 years). Epidemiological data was reviewed, as well as treatment

modalities and survival. Out of the 14 patients, $6(42 \%)$ had tumor in the mandible, while $8(58 \%)$ had it in the maxilla. The histological types found were: chondroblastic, osteoblastic, fibroblastic and one similar to malignant fibrous histiocytoma. High pathological grade (3 or 4) was detected in 13 cases, while only one mandibular case had low pathological grade. All patients underwent surgical resection and immediate reconstruction. Adjuvant therapy included postoperative radiation and post- and preoperative chemotherapy.

Chindia et al. ${ }^{11}$ reported 14 cases of osteosarcomas of the maxillary bones, being 11 in the maxilla, 2 in the mandible and 1 in the zygomatic arch. Patients' ages ranged from one week to 50 years old (mean age of 29.7 years), equally distributed between genders. The most common clinical aspects were pain and fast volume increase, while the radiographic and histological aspects were considerably diverse. The authors reported that at least 6 of the patients who were followed up from 2 to 6 months had extensive recurrences that led to death. Treatment approaches were chemotherapy, radiotherapy and surgery, isolated or in combination. A retrospective review of clinical, radiographic and histopathological data of 25 patients was conducted with the purpose of comparing the clinical behavior of tumors and analyzing the differences reported for tumors in other sites4. Mean age at presentation of primary lesions was 36.9 years (10-87 years), with slight prevalence of females. The most common aspects of presentation were: tumor volume increase, pain, ulceration and neurological disorder; radiographic aspects showed radiopaque and radiolucent areas. Histologically, there was immature bone trabeculae, which was separated by a stroma that cytologically ranged from low to high grade. In some areas, marked atypia and mitotic activity could be observed. Most lesions had areas of chondroid formation, in addition to neoplastic osteoid formation. The main complication was local recurrence. Metastases were rare and occurred isolated or at a late stage in disease progression.

Mandibular osteosarcomas have a better prognosis than maxillary osteosarcomas. ${ }^{12}$ Unni and Dahlin described that osteosarcomas of jaw are usually histologically grade II and occasionally grade III, and they are associated with a better prognosis than conventional osteosarcoma. ${ }^{13}$ In our case the 
tumor was grade III and more aggressive and present with lung metastasis. Occasionally craniofascial osteosarcoma may present with aggressive behavior, for which aggressive management protocol should considered, ${ }^{4}$ like in our case.

Takahama Junior et al. ${ }^{5}$ have recently evaluated the clinical and pathological aspects and the immunohistochemical expression of p53, MDM2, PCNA, and KI67 proteins in 25 cases of OS of the head and neck.

Pulmonary metastases occurs in approximately half of the osteosarcoma patients and $30 \%$ to $50 \%$ of the osteosarcoma patients still die of pulmonary metastasis. In the craniofascial osteosarcoma, lung metastasis were very rare and have very good prognosis as compared to long bone osteosarcoma. ${ }^{14}$ The mean age of patients was 29 years and the most common site was the mandible (60\%). The predominant histological type was chondroblastic (72\%). The immunohistochemical analysis was positive in $52 \%$ of cases for p53, 24\% for MDM2, 84\% for CDK4, 92\% for PCNA and $88 \%$ for KI67. Most patients were treated with surgery alone or in association with chemotherapy. Five year and 10 -year survival rates were $59 \%$ and $49 \%$, respectively, and the most important prognostic factors were previous exposure to radiation and osteoblastic histological type.

The most common clinical presentation of osteosarcoma is pain in the involved region of bone, with or without a soft tissue mass. The lesions are slightly more common in men. ${ }^{15}$ In our case the child present with non pain swelling over mandible and soft tissue extension.

In the case presented in our study, being reported due to its rarity in both age of presentation and its aggressiveness.

\section{References}

[1] Neville BW, Damm DD, Allen CM, Bouquot JE. Patologia oral e maxilofacial. Rio de Janeiro: Guanabara Koogan; 2004. p. 534-8.

[2] Zarbo RJ. In: Regezi JA, Sciuba JJ. Patologia bucal:correlações clinicopatológicas. 3 ed. Rio de Janeiro: Guanabara Koogan 2000. p.475.

[3] Smith RB, Apostolakis LW, Karnell LH, Koch BB, Robinson $\mathrm{RA}$, Zhen W, et al. National cancer data base report on osteosarcoma of the head and neck. Cancer 2003;98:1670-80.

[4] Alberto De Biase a, Walter Morciano a, Francesco Carpino b, Michele Milella c:Aggressive chondroblastic osteosarcoma of the jaw bone. Oral Oncology EXTRA $2005 ; 41: 296-98$.

[5] Takahama Júnior A, Alves FA, Pinto CAL, Carvalho AL, Kowalski LP, Lopes MA. Clinicopathological and immunohistochemical analysis of twenty-five head and neck osteosarcomas Oral Oncol 2003; 39 (5): 521-30.

[6] Sturgis EM, Potter BO. Sarcomas of the head and neck region. Curr Opin Oncol 2003;15(3):239-52.

[7] Patel SG, Meyers P, Huvos AG, Wolden S, Singh B, Shaha $\mathrm{AR}$, et al. Improved outcomes in patients with osteogenic sarcoma of the head and neck. Cancer 2002;95 (7):1495-503.

[8] Kassir RR, Rassekh CH, Kinsella JB, Segas J, Carrau RL, Hokanson JA. Osteosarcoma of the head and neck: metaanalysis of non-randomized studies. Laryngoscope 1997;107: 56-61.

[9] Ha PK, Eisele DW, Frassica FJ, Zahurak ML, McCarthy EF. Osteosarcoma of the head and neck: a review of the Johns Hopkins experience. Laryngoscope 1999;109:964-69.

[10] Mardinger O, Givol N, Talmi Y.P, Taicher S, Saba K. Osteosarcoma of the jaw Oral Med Oral Pathol Oral Radiol Endod 2001; 91: 445-51.

[11] Chindia ML, Guthua SW, Awange DO, Wakoli KA Osteosarcoma of the maxillofacial bones . Kenyans $\mathrm{J}$ Maxillofac Surg 2002; 26 (2): 98-101.

[12] Garrington GE, Scofield HH, Cornyn J, Hooker SP.1967Osteosarcoma of the jaws. Analysis of 56 cases. Cancer 1967;20: 377-391.

[13] Unni KK, Dahlin DC (1984) Grading of bone tumors. Semin Diagn Pathol 1984;1: 165-172.

[14] Glasser DB, Lane JM, Huvos AG, Marcove RC, Rosen G. Survival, prognosis, and therapeutic response in osteogenic sarcoma. The Memorial Hospital experience. Cancer. 1992;69:698-708.

[15] Bennett JH, Thomas G, Evans AW, Speight PM. Osteosarcoma of the jaw: A 30 years retrospective review. Oral Surg Oral Med Oral Pathol Oral Radiol Endod 2000;90: 323-332. 